\title{
Muscle specific miRNAs are induced by testosterone and independently upregulated by age
}

\author{
Søren Nielsen ${ }^{1}$ *, Thine Hvid ${ }^{1}$, Meghan Kelly ${ }^{1}$, Birgitte Lindegaard ${ }^{1}$, Christine Dethlefsen ${ }^{1}$, \\ Kamilla Winding ${ }^{1}$, Neha Mathur ${ }^{1}$, Camilla Scheele ${ }^{1}$, Bente K. Pedersen ${ }^{1}$ and Matthew J. Laye ${ }^{1,2}$ \\ Department of Infectious Diseases, The Centre of Inflammation and Metabolism and the Centre for Physical Activity Research, Rigshospitalet, University of \\ Copenhagen, Copenhagen, Denmark \\ 2 The Buck Institute for Research on Aging, Novato, CA, USA
}

Edited by:

Wataru Aoi, Kyoto Prefectural

University, Japan

Reviewed by:

John J. McCarthy, University of

Kentucky, USA

Aaron P. Russell, Deakin University,

Australia

*Correspondence:

Søren Nielsen, The Centre of

Inflammation and Metabolism and

The Centre for Physical Activity

Research, Rigshospitalet 7641,

Blegdamsvej 9, DK-2100

Copenhagen, Denmark

e-mail:soren_nielsen@

inflammation-metabolism.dk
Age dependent decline in skeletal muscle function leads to impaired metabolic flexibility in elderly individuals. Physical activity and testosterone treatment have proven efficient strategies for delaying this condition. However, a common molecular pathway has not been identified. Muscle specific miRNAs (myomiRs) regulate metabolic pathways in skeletal muscle, are regulated by physical activity, and have response elements for testosterone in their promoter region. We therefore hypothesized that myomiRs would be regulated in skeletal muscle during aging. We further investigated any potential gender-dependent regulation of these miRNAs. We found that the myomiRs miR-1, miR-133a, and miR-133b were increased in skeletal muscle of elderly men compared to younger men. In addition, miR-133a/133b expression was markedly higher in women compared to men. Elimination of circulating testosterone in men was associated with lower levels of miR-133a and miR-133b. A positive regulatory effect of testosterone on miR-133a/133b expression was confirmed in castrated male C57BL/6J mice and in a model of primary human myocytes. Yet, an improvement of fitness level in the testosterone depleted men resulted in a down-regulation of miR133a/b. In conclusion, alterations in fitness level and circulating testosterone seem to represent two independent regulatory events where testosterone is a specific regulator of miR-133a/b expression.

Keywords: miRNA, skeletal muscle, myomiRs, aging, gender, exercise

\section{INTRODUCTION}

The normal aging process involves a decline in skeletal muscle function. Several metabolic alterations are associated with aging, including impaired glucose, lipid and protein metabolism, subsequently leading to metabolic inflexibility and loss of skeletal muscle function (Ramamani et al., 1999; Gibney et al., 2005; Frederiksen et al., 2012).

Age related metabolic dysregulation is a multifactorial and gender specific process, involving decreased physical activity and alterations in steroid hormonal levels (Booth and Zwetsloot, 2010; Kelly and Jones, 2013). Importantly, physical activity delays and/or diminishes the age related metabolic alterations independently of steroid hormones (Yarasheski, 2003). The steroid hormone testosterone is synthesized mainly in the testis in men and to a minor degree in the ovaries in women and plays a key role in carbohydrate, fat, and protein metabolism in tissues expressing the androgen receptor (AR), including skeletal muscle (Ramamani et al., 1999; Frederiksen et al., 2012; Phillips et al., 2012). In men, circulating testosterone levels peak around age $30-40$ and gradually decrease thereafter. At the age of 75 , testosterone levels are approximately decreased by $30 \%$, compared to peak levels (Deslypere and Vermeulen, 1984; Muller et al., 2003). In adult women, circulating testosterone concentrations are approximately 10 times as low as in men (Torjesen and Sandnes, 2004) and likewise decrease with age (Yasui et al.,
2012). Circulating testosterone can be bound to carrier proteins but also exist in an unbound form, which is normally referred to as free testosterone. The molecular mechanisms by which circulating testosterone controls muscle metabolism are not fully clear.

The canonical pathway through which free testosterone acts is by diffusing through the cell wall and binding to cytosolic AR's, which translocate to the nucleus and bind to DNA as dimers. Nuclear AR binds to androgen response elements (AREs) within genomic promoter regions, where it can regulate gene transcription (Heemers and Tindall, 2007). Interestingly, AREs have been identified in the promoter regions of the non-coding regulatory group of RNAs called microRNAs (miRNAs) (Delic et al., 2010). Mature miRNAs are typically 20-22bp in length, and function through physical association with the $3^{\prime} \mathrm{UTR}$ of a mRNA, either degrading the mRNA or causing ribosomal stalling to reduce protein translation (Bartel, 2004). A single miRNA can regulate the expression of hundreds of mRNAs and proteins (Lim et al., 2005; Baek et al., 2008) and these small RNAs therefore play a crucial role in many aspects of biology, including cell cycle regulation, developmental processes and metabolic regulation (Chen et al., 2006; Zhao et al., 2007; Gallagher et al., 2010; Lujambio and Lowe, 2012). miR-1, miR-133a, miR-133b, and miR-206 belong to a group of muscle specific miRNAs (myomiRs) crucial for the regulation of skeletal muscle development and function (Chen et al., 
2006; van Rooij et al., 2008). Five active ARE motifs have been experimentally identified and verified in the promoter region near the co-transcribed miR-206/miR-133b locus (Wyce et al., 2010). ARE motifs near the miR-1/miR-133a loci, have not yet been identified. Importantly, in addition to steroid hormones, myomiR loci are regulated by muscle specific transcription factors referred to as Myogenic Regulatory Factors (MRFs) (Chen et al., 2006; Rao et al., 2006; Sweetman et al., 2008). MRFs are vital in the process of coordinating skeletal muscle development and repair (Ramamani et al., 1999). Interestingly, AREs are also found within the promoters for two of the four MRFs, myogenic differentiation 1 (MYOD1) and myogenin (MYOG), suggesting that androgens may indirectly alter myomiR transcription (Dubois et al., 2012). Taken together, transcriptional regulation of myomiRs might be controlled directly and/or indirectly by androgens.

We have previously shown that the expression of all four myomiRs decreases in response to an endurance training program suggesting that these myomiRs are important for metabolic adaptation to endurance exercise (Nielsen et al., 2010). Furthermore, a study by Drummond and co-workers examined myomiR expression patterns in muscle in relation to aging (Drummond et al., 2008b). No differences in basal expression of mature functional myomiRs between elderly and younger men were detected. However, expression of myomiRs in response to resistance exercise and ingestion of essential amino acids was different between elderly and young men. Therefore, anabolic resistance in aged skeletal muscle seems to have a different response mechanism to anabolic stimuli. In addition, myomiR expression is altered in several mice and human models investigating skeletal muscle atrophy and hypertrophy (Huang et al., 2011; Ringholm et al., 2011) and miR-206 is specifically down-regulated in muscle of patients with Amyotrophic lateral sclerosis (Russell et al., 2012).

Interestingly, one validated target of $\mathrm{miR}-133 \mathrm{a} / \mathrm{b}$ is the insulinlike growth factor-1 receptor (IGF-1R) in skeletal muscle (Huang et al., 2011), making miR-133a/b a likely regulator of growth factor signaling through the AKT signaling pathway (Schiaffino and Mammucari, 2011). Furthermore, it has been shown that miR-133a and miR-206 are lower in skeletal muscle of people with type 2 diabetes (Gallagher et al., 2010). Therefore, several pieces of evidence indicate that myomiRs are likely to be involved in the process of maintaining metabolic flexibility in skeletal muscle.

Thus, our aim was to investigate whether myomiRs are dysregulated in aged skeletal muscle. Based on previous observations that myomiR expression decreases with increased aerobic fitness and that aerobic fitness decreases with age (Booth and Zwetsloot, 2010; Nielsen et al., 2010; Keller et al., 2011), we hypothesized that skeletal muscle of older individuals would have higher myomiR expression. In addition, given that men and women have markedly different testosterone levels and given a recent report showing that the transcriptome in aged muscle differs markedly between genders (Liu et al., 2013), we further investigated potential differences in myomiR expression between men and women. Finally, we examined whether testosterone, a hormone delaying the age dependent decline in muscle function, regulates myomiR expression.

\section{METHODS \\ ETHICAL APPROVAL}

The human studies (in vivo study 1 and 2) were approved by the local Ethical Committee of Copenhagen and Frederiksberg (KF 01-141/04) (H-4-2009-102) and were performed in accordance with the Declaration of Helsinki. The purpose of the study and its possible risks and discomforts were explained to the participants before their written consent was obtained.

\section{IN VIVO STUDY 1}

Nine older men and nine older women participated in the study. Before inclusion in the study, a medical examination with blood test screening, maximal oxygen consumption test $\left(\mathrm{VO}_{2} \mathrm{max}\right)$, and oral glucose tolerance test were performed. Nine younger men and nine younger women with similar body mass indexes (BMIs) as the elderly were recruited as control groups. The control group underwent the same examinations and tests as the two older groups before inclusion. Subjects were excluded from the study if they had any infections 2 weeks before the study or if they were diabetic or had impaired glucose tolerance. Subject characteristics are listed in Table 1. All subjects arrived in the morning in a fasted state to the lab.

\section{IN VIVO STUDY 2}

Eight men with prostate cancer undergoing medical clinical castration, in form of the LHRH-agonist, Goserelin (Zoladex) were recruited from the Departments of Urology at Rigshospitalet and Herlev Hospital, as previously described (Hvid et al., 2013). Briefly, all subjects were treated with Goserelin for at least 3 months before inclusion in the study and continued the treatment throughout the study. Subjects were excluded if they had any infections 2 weeks before the study started or if they had severe health threatening metastases. Eight healthy men with normal testosterone levels, matched for age and $\mathrm{VO}_{2} \max$, were recruited as a control group. Subject characteristics are listed in Table 2. On the experimental day subjects arrived in the morning in a fasted state. Testosterone levels were measured before an oral glucose tolerance test, $3-5$ days pre and post-training.

\section{TRAINING PROTOCOL IN VIVO STUDY 2}

Subjects from in vivo study 2 performed and endurance training program for 12 weeks on a cycle ergometer (Monark 839E, Monark Ltd, Varberg, Sweden) consisting of three training sessions per week. All training sessions consisted of $5 \mathrm{~min}$ of warm up, $35 \mathrm{~min}$ of interval training and $5 \mathrm{~min}$ of cool down, as previously described (Hvid et al., 2013). The first 6 weeks the mean training intensity was at $65 \%$ of $\mathrm{VO}_{2}$ max, and the last 6 weeks at $75 \%$ of $\mathrm{VO}_{2}$ max. All training sessions were supervised and heart rate was continuously monitored (RS400; Polar, Kempele, Finland). The subjects were instructed to maintain their habitual diet and level of physical activity during the study. The subjects completed the diet and activity records for 3 days in the beginning of the training period and for 3 days at the end of the training period. Diet did not differ between groups.

\section{BODY COMPOSITION}

Whole body fat and fat-free tissue mass measurements were performed using a dual-energy X-ray absorptiometry (DXA) scanner 
Table 1 | In vivo study 1.

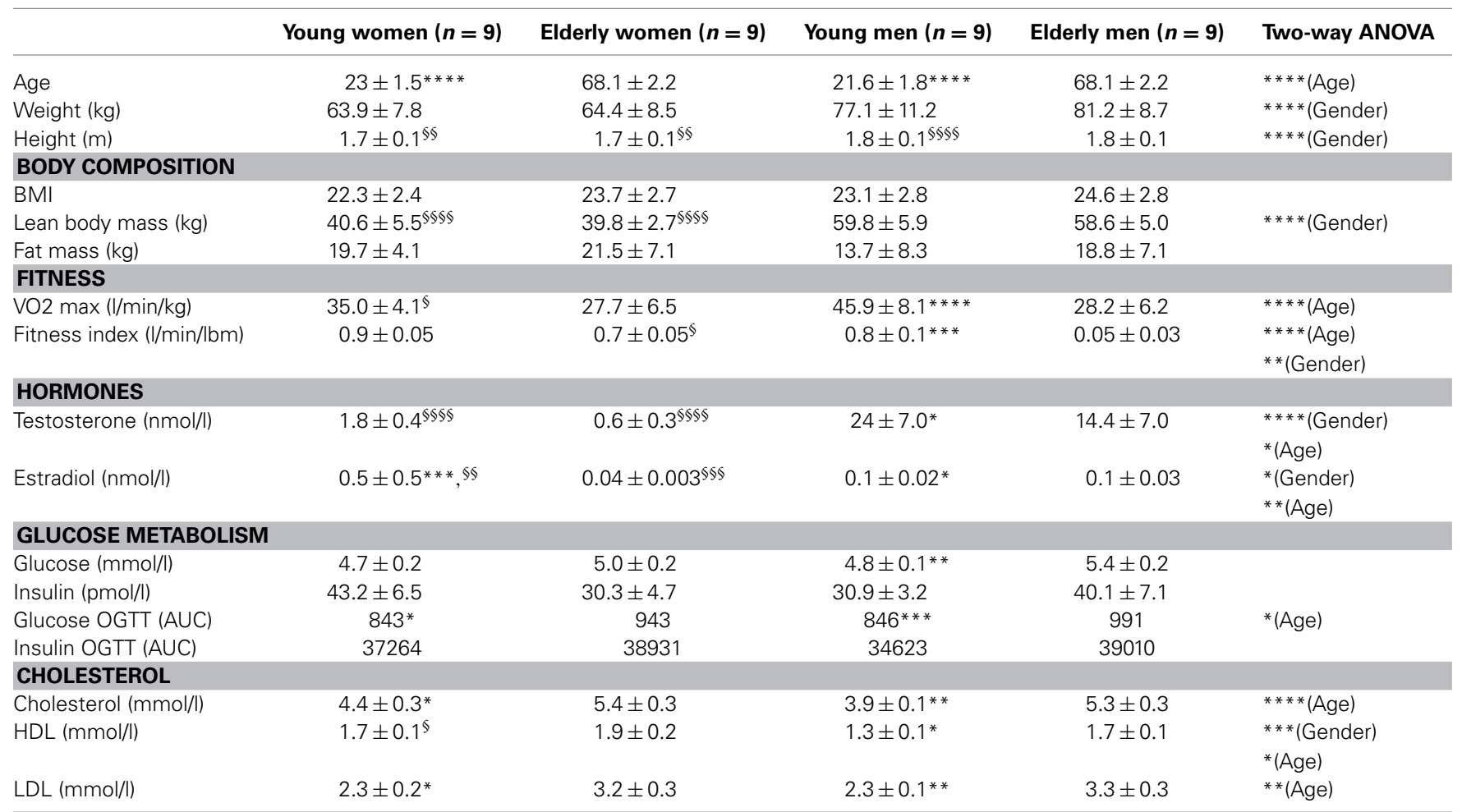

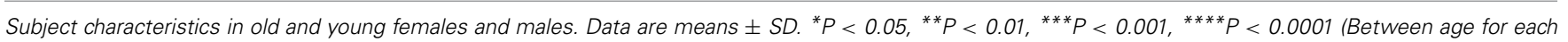

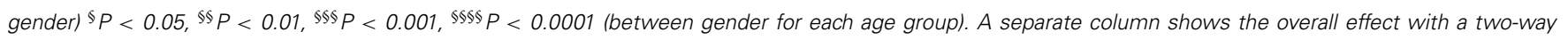
ANOVA (same significance levels as between age).

(Lunar Prodigy, GE Healthcare, WI, Madison USA, software v. 8.8 ) in both human in vivo studies.

\section{MUSCLE BIOPSIES}

Muscle biopsies from vastus lateralis were obtained at rest in the fasted state using the percutaneous needle method with suction under local anaesthesia, using 3-5 ml of $20 \mathrm{mg} \mathrm{ml}^{-1}$ lidocaine (SAD, Denmark Copenhagen). Muscle tissue was immediately frozen in liquid nitrogen and stored at $-80^{\circ} \mathrm{C}$ until further analysis.

\section{ANIMAL EXPERIMENTAL PROTOCOLS}

Nine male 7 weeks old wild-type C57BL/6J mice originally obtained from Taconic, Denmark and inbred at the animal facility (Copenhagen, Denmark). All experiments were approved by The Animal Experiments Inspectorate of Denmark in accordance with the National Health and Medical Research Council of Australia Guidelines on Animal Experimentation. Mice were maintained on a 12-h light, 12-h dark cycle on standard rodent chow diet. Eight weeks old mice ( $n=5$ ) were surgically castrated, eliminating circulating testosterone. The control mice $(n=4)$ were sham operated to obtain equal treatment between groups. Twenty-four weeks post castration and sham operation the mice were sacrificed and quadriceps muscle was snap frozen in liquid nitrogen and stored at $-80^{\circ} \mathrm{C}$ until further use. Quadriceps muscle was powdered in liquid nitrogen before RNA isolation (see separate procedure description).

\section{CELL EXPERIMENTS}

Satellite cells were isolated from muscle biopsies as previously described (Scheele et al., 2012). Unless otherwise stated, all cell culture reagents were from Invitrogen, Carlsbad, CA, USA. Briefly, a muscle biopsy was obtained from vastus lateralis and digested in $5 \mathrm{ml} \mathrm{HamF10} \mathrm{media} \mathrm{containing} \mathrm{50 \%} \mathrm{trypsin} \mathrm{EDTA}$ $(1 \times)$ and $5 \mathrm{mg}$ collagenase IV (Sigma-Aldrich, St. Louis, MO, USA) and BSA (Sigma-Aldrich) at $37^{\circ} \mathrm{C}$ for $5 \mathrm{~min}$. The digestion solution was inactivated in serum on ice and the procedure was repeated. The digestion solution was filtered and centrifuged at $800 \mathrm{~g}$ for $7 \mathrm{~min}$. Following washing with HamF10, cells were pre-plated in a $60 \mathrm{~mm}$ culture plate, to diminish fibroblast contamination. After $3 \mathrm{~h}$ incubation, the cell suspension was transferred to a $25 \mathrm{~cm}^{3}$ flask coated with matrigel (BD, Franklin Lakes, NJ, USA). The media, HamF10, 20\% FBS, 1\% Penicillin/Streptomycin and 1\% Fungizone, was changed on day 4 after isolation and thereafter every second day. Cell cultures were expanded from the $25 \mathrm{~cm}^{3}$ flask to a $10 \mathrm{~cm}$ plate, which were assigned passage (P) 1. Thereafter, cells were split 1:3 at $75 \%$ confluence. Experiments were performed on cells at P6-P7. For differentiation, cells were seeded in DMEM low glucose $(1 \mathrm{~g} / \mathrm{L}), 10 \%$ FBS, 1\% Penicillin/Streptomycin and, upon confluence, media was changed to DMEM high glucose $(4.5 \mathrm{~g} / \mathrm{L}), 2 \%$ horse serum, $1 \%$ Penicillin/Streptomycin. Experiments were performed on day 6 of differentiation by which time cells had fused into polynucleated myotubes. Differentiated myotubes were then incubated with $100 \mathrm{nM}$ free testosterone 99\% purum (Sigma-Aldrich, 
Table 2 | In vivo study 2.

\begin{tabular}{|c|c|c|}
\hline & $\begin{array}{l}\text { Control } \\
(n=8)\end{array}$ & $\begin{array}{l}\text { LHRH-agonist } \\
\qquad(n=8)\end{array}$ \\
\hline Age & $67.5 \pm 2.9$ & $67.9 \pm 6.8$ \\
\hline Weight (kg) & $80.5 \pm 12.7$ & $80 \pm 13.8$ \\
\hline Height (cm) & $177.6 \pm 4.5$ & $176.1 \pm 9.4$ \\
\hline \multicolumn{3}{|l|}{ BODY COMPOSITION } \\
\hline BMI & $25.5 \pm 3.3$ & $25.7 \pm 3.6$ \\
\hline Lean body mass pre-training $(\mathrm{kg})$ & $57.27 \pm 2.1$ & $52.15 \pm 2.5$ \\
\hline Lean body mass post-training $(\mathrm{kg})$ & $57.32 \pm 2.07$ & $52.13 \pm 2.4$ \\
\hline Fat mass pre-training $(\mathrm{kg})$ & $20.2 \pm 2.9$ & $24.5 \pm 3.8$ \\
\hline Fat mass post-training $(\mathrm{kg})$ & $19.3 \pm 2.7$ & $23.19 \pm 2.9$ \\
\hline \multicolumn{3}{|l|}{ FITNESS } \\
\hline VO2 max pre-training $(1 / \mathrm{min} / \mathrm{kg})$ & $27 \pm 3.8$ & $25.3 \pm 4.4$ \\
\hline VO2 max post-training (I/min/kg) & $31.3 \pm 3.3^{* * * *}$ & $29.4 \pm 3.9 * * * *$ \\
\hline \multicolumn{3}{|l|}{ HORMONES } \\
\hline Testosterone (nmol/l) & $13.5 \pm 5.1^{15 s}$ & $0.5 \pm 0.4$ \\
\hline
\end{tabular}

Subject characteristics in LHRH-agonist treated prostate cancer patients and healthy controls. Fitness, lean body mass and fat mass significance levels were calculated with a Two-Way ANOVA RM and bonferroni's multiple comparison test was used as post-hoc test to identify any differences between groups before and after training. ${ }^{* * *} P<0.0001$, symbolize overall effect of training. The remaining significance levels were calculated with unpaired t-tests. ${ }^{\$ \$} \$ P<0.001$ (between the two groups). Data are means $\pm S D$.

Switzerland) and $5 \alpha$-androstan-17 $\beta$-ol-3-one (Sigma-Aldrich, Switzerland) for $24 \mathrm{~h}$ on FBS free low glucose DMEM media to avoid contamination of testosterone present in the FBS. Testosterone and $5 \alpha$-androstan-17 $\beta$-ol-3-one were dissolved in Dimethyl Sulphoxide (DMSO) (Sigma-Aldrich, Switzerland) and thereafter mixed with DMEM media. The vehicle, DMSO, was added in equal concentrations as the testosterone stimulated cultures. All cell culture experiments were repeated 4 times $(n=4)$.

\section{RNA ISOLATION}

Approximately $30 \mathrm{mg}$ skeletal muscle samples, powdered quadriceps muscle (mouse study) or Vastus Lateralis (human studies) were homogenized in TRIzol (Life Technologies) using a Tissuelyser (Qiagen, Valencia, CA, USA) and total RNA was isolated according to the manufacturer's protocol. Total RNA was dissolved in RNase-free water and quantified using a Nanodrop ND 1000 (Thermo Scientific, Wilmington, DE, USA).

\section{myomir QUANTIFICATION}

The abundance of mature miRNAs was measured using TaqMan miRNA assays specific for miR-1 (Assay ID: 002222), miR133a (Assay ID: 002246), miR-133b (Assay ID: 002247) and -206 (Assay ID: 000510) according to the manufacturer's directions (Applied Biosystems Inc., Foster City, CA, USA). Briefly, reverse transcription was performed with miRNA-specific RT primers and $10 \mathrm{ng}$ of total RNA for $30 \mathrm{~min}$ at $37^{\circ} \mathrm{C}$ followed by $10 \mathrm{~min}$ incubation at $95^{\circ} \mathrm{C}$. Quantitative real-time PCR (qPCR) was performed using the ViiA7 Sequence Detection System (Applied Biosystems) according to the manufacturer's protocol in triplicates, using the RT product and miRNAspecific PCR primers for 40 cycles. The final miRNA specific primer assay volume was $5 \%(20 \times)$ of the total volume for the qPCR, as recommended by the manufacturer's protocol (Applied Biosystems). RNU48 (Assay ID: 001006), a small nuclear RNA, and $18 \mathrm{~S}$ was measured using the same type of detection assay (Applied Biosystems), and was used as an endogenous control for miRNA expression analyses in the human in vivo studies. Endogenous controls were tested for systematically variation between samples before further analysis. In the mouse study 18S (Mm03928990_g1) was used as an endogenous control, as RNU48 is not expressed in mice. RNU48 was used as endogenous control in human in vivo study 2 and in the human primary myotube in vitro study. In the human in vivo study $1,18 \mathrm{~S}$ expression was used as an endogenous control due to differentially expression of RNU48 between elderly and young subjects. Expression levels were calculated with the standard curve method. PCR efficiencies, linearity slopes, cycle threshold ranges and concentrations for the qPCR primers used in the study are listed in Table 3.

\section{MRNA OUANTIFICATION}

Total RNA $(0.5 \mu \mathrm{g})$ was reverse-transcribed using cDNA high capacity kit (Applied Biosystems) according to the manufacturer's protocol. cDNA samples were loaded in triplicate and $\mathrm{qPCR}$ was performed using ViiA7 Sequence Detection system (Applied Biosystems, Foster City, CA, USA) according to the manufacturer's protocol using either SYBR chemistry or primer-probe based pre-optimized detection methods. Primers were designed using Roche Applied Science Assay Design Center (Roche).

\section{Human primers}

AR (CT-range: 23.1-24.5)

Pre-optimized assay from Applied Biosystems (Hs00171172_m)

MYOD (CT-range: 24.2-26.2)

Forward primer-CACTACAGCGGCGACTCC

Reverse primer-TAGGCGCCTTCGTAGCAG

MYOG (CT-range: 21.9-23.9)

Forward primer-GCTCAGCTCCCTCAACCA

Reverse primer-GCTGTGAGAGCTGCATTCG

MYF5 (CT-range: 24.7-26.8)

Reverse primer-CTATAGCCTGCCGGGACA

Reverse primer-TGGACCAGACAGGACTGTTACAT

\section{Mouse primers}

Ar (CT-range: $21.3-23.2$ )

Forward primer-TCGGTGGAAGCTACAGACAA

Reverse primer-TAGACCCTTCCCAGCCCTAA

Myog (CT-range: 24.1-27.2)

Forward primer-GGTGCCCAGTGAATGCAACT

Reverse primer-AGCCGCGAGCAAATGATCT

Myod (CT-range: 22.6-25.2)

Forward primer-CACTCCGGGACATAGACTTGACA

Reverse-CCCCGACGGCTCTCTCTG 
Table 3 | PCR standard curve data.

\begin{tabular}{|c|c|c|c|c|c|c|c|c|}
\hline \multirow[b]{2}{*}{ Primers } & \multicolumn{4}{|c|}{ In vivo study 1 (Figure 1) } & \multicolumn{4}{|c|}{ In vivo study 2 (Figure 3 ) } \\
\hline & Lin. slope & Efficiency (\%) & Range (CT) & $\begin{array}{l}\text { Primer } \\
\text { conc. }\end{array}$ & Lin. slope & Efficiency (\%) & Range (CT) & $\begin{array}{l}\text { Primer } \\
\text { conc. }\end{array}$ \\
\hline miR-1 & -3.12 & 106 & $18.3-22.3$ & \multirow{3}{*}{$20 x$} & -3.55 & 94 & $21.6-24.9$ & \multirow{3}{*}{$20 x$} \\
\hline miR-133b & -3.49 & 95 & 19.6-22.9 & & -3.27 & 102 & $19.7-22.7$ & \\
\hline miR-206 & 3.5 & 95 & $21.6-24.6$ & & -3.21 & 104 & $23.2-26.2$ & \\
\hline MYOG & - & - & - & - & -3.15 & 105 & $22.7-26.5$ & \multirow{4}{*}{$\begin{array}{l}\mathrm{F}: 300 \mathrm{nM} \\
\mathrm{R}: 300 \mathrm{nM}\end{array}$} \\
\hline MYF-5 & - & - & - & - & -3.28 & 101 & $25.5-29.3$ & \\
\hline AR & - & - & - & - & -3.51 & 95 & $23.7-28.0$ & \\
\hline $18 \mathrm{~S}$ & -3.26 & 102 & $14.7-18.5$ & $\begin{array}{l}\text { F: } 300 \mathrm{nM} \\
\mathrm{R}: 300 \mathrm{nM}\end{array}$ & -3.44 & 97 & $13.0-17.2$ & \\
\hline miR-206 & -3.41 & 97 & $24.4-27.5$ & $20 \mathrm{X}$ & -3.49 & 95 & $22.9-27.1$ & \multirow{2}{*}{$20 x$} \\
\hline RNU48 & - & - & - & - & -3.16 & 105 & $26.8-30.7$ & \\
\hline MYOD/Myod & -3.25 & 102 & $22.8-26.8$ & \multirow{5}{*}{$\begin{array}{l}F: 300 n M \\
R: 300 n M\end{array}$} & -3.47 & 96 & $22.7-26.9$ & \multirow{5}{*}{$\begin{array}{l}\mathrm{F}: 300 \mathrm{nM} \\
\mathrm{R}: 300 \mathrm{nM}\end{array}$} \\
\hline MYOG/Myog & -3.83 & 88 & $25.3-29.1$ & & -3.36 & 99 & $20.5-24.5$ & \\
\hline MYF-5/Myf-5 & -3.8 & 84 & $26.1-31.0$ & & -3.53 & 94 & $22.5-26.8$ & \\
\hline $\mathrm{AR} / \mathrm{Ar}$ & -3.19 & 104 & $22.9-25.8$ & & -3.46 & 96 & $27.1-31.2$ & \\
\hline $18 \mathrm{~S}$ & -3.36 & 99 & $13.4-17.4$ & & -3.5 & 95 & $13.7-18.1$ & \\
\hline
\end{tabular}

PCR efficiency, linearity slope, cycle threshold range and concentration for each primer used in the study.

Myf5 (CT-range: 26.3-29.1)

Forward primer-GCCAGTTCTCCCCTTCTGAGTS

Reverse primer-ACTGGTCCCCAAACTCATCCT

Pre-optimized assays from Applied Biosystems for 18S rRNA (Human, Hs99999901_s1. Mouse, Mm03928990_g1) was used as endogenous control. To assess amplification efficiency, two fold serial dilutions were performed for all primers. Based on these, cDNA was diluted 1:10 for Myog, MyoD, Myf-5, and Ar and 1:200 for $18 \mathrm{~S}$, to ensure linear amplification. Melting curve analysis was performed to ensure primer specificity for SYBR green reactions. Expression levels were calculated with the standard curve method (Table 3).

\section{Statistical analysis}

The statistical analysis was performed using GraphPad Prism 6.0 (GraphPad Software Inc., La Jolla, CA, USA). Results in the figures are presented as means \pm SEM. Data in the tables are presented as means $\pm \mathrm{SD}$. All data were tested for normality of distribution before further analysis using the KolmogorovSmirnov test. An ordinary Two-Way ANOVA model 1 was used to analyse the data in Figure 1 and Table 1 (age and gender as main effects) and Two-Way ANOVA repeated measures was used for Figure 3. Bonferroni's multiple comparisons test was used as post-hoc tests to identify differences expression within the two gender groups when the Two-Way ANOVA tests reached significant level $P<0.05$. Unpaired $t$-tests were used to analyse the data in the mice study (Figure 4), the cell culture study (Figure 5) and the pre-training expression levels of the myogenic markers (MYOD, MYOG, MYF5) and the androgen receptor (AR) (Figure 3). Linear regression analysis was applied as stated in the legends Figure 2, $P<0.05$ was used as significance level.

\section{RESULTS}

\section{GENDER AND AGE AFFECTS miR-133a AND miR-133b EXPRESSION}

To examine whether age and/or gender affects myomiR expression in skeletal muscle, we measured myomiRs at rest in biopsies taken from vastus lateralis in young and elderly men and women (Figure 1). An ordinary Two-Way ANOVA revealed a markedly effect of both gender and age on miR-133a and miR-133b expression $(P<0.01)$, where both factors are associated with an overall higher expression of both mature miRNA transcripts. Where no effect of age and/or gender on miR-1 and miR-206 expression was detected $(P>0.05)$, a significant interaction on miR-1 expression was identified with a Two-Way ANOVA test $(P<0.05)$. As expected, muscle mass was clearly lower in the women (Table 1). Interestingly, an association between increased muscle mass and downregulation of myomiRs has been reported (McCarthy and Esser, 2007). Other variables that might be related to myomiR expression included aerobic fitness and testosterone levels, which differed between the four groups (Table 1) and were therefore selected for additional examination.

\section{miR-1, miR-133a AND miR-133b EXPRESSION IS UPREGULATED WITH AGING IN MEN}

To address a potential involvement of miR-1, miR-133a, and miR-133b in the age-related decline in muscle function for both genders, we used a bonferroni multiple comparison post-hoc test. 


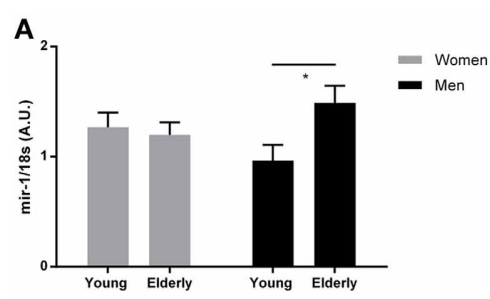

C

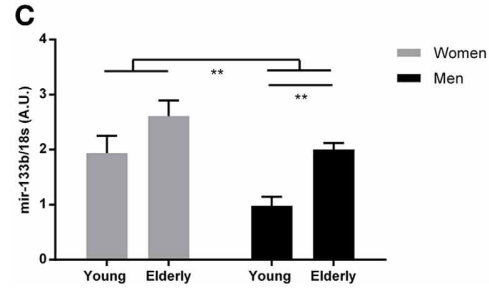

FIGURE 1 | MyomiR expression in human skeletal muscle from elderly and younger individuals. Muscle biopsies were obtained from a cohort of healthy elderly and younger men and women ( $n=9$ in each group) and myomiR levels were measured by using qPCR. An ordinary Two-Way ANOVA revealed an overall effect of both gender and age on miR-133a and miR-133b expression $(\mathbf{B}, \mathbf{C})(* * P<0.01)$ and a significant interaction for

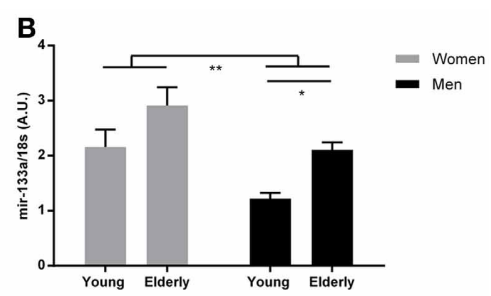

D

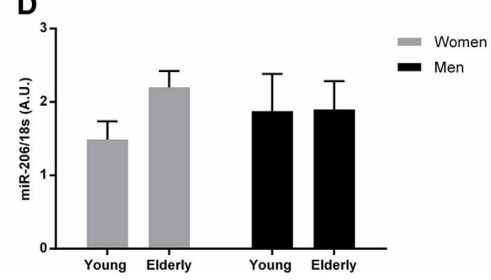

miR-1 expression (** $P<0.01)$. When using Bonferroni multiple comparison post-hoc test it was demonstrated that miR-1 (A), miR-133a (B), and miR-133b (C) expression levels were higher in elderly compared to younger men $\left(* P=0.02,{ }^{*} P=0.03,{ }^{* * *} P=0.008\right.$, respectively) There was no effect of age or gender on mir-206 expression (D) $(P>0.05)$. Data are mean \pm SEM.

12 week aerobic exercise training programme to test if increased fitness level altered myomiR expression independent of plasma testosterone levels. Muscle biopsies were obtained at rest before and after the training period. Partly in line with our previous results (Nielsen et al., 2010), a Two-Way ANOVA (RM) demonstrated a main effect of training in terms of decreased expression in all four myomiRs (miR-1, $P<0.0001$. miR-133a, $P<$ 0.01. miR-133b, $P<0.0001$, miR-206 $P<0.05)$. Surprisingly, a bonferroni multiple comparison test revealed reduced miR$133 \mathrm{a} / \mathrm{b}$ expression (miR-133a, $P=0.02$. miR-133b, $P=0.03$.) before training in the LHRH-agonist treated participants with almost no plasma testosterone (Figures 3B,C). That observation was in opposition to the association observed in the elderly vs. young participants in study 1 (Figures 2F,G). miR-1 and miR-206 expression were not altered by LHRH-agonist treatment before the training period $(P>0.05)$ (Figures 3A,D). Regardless of preexercise intervention level, training equalized the expression of miR-133a and miR-133b between healthy and LHR-treated participants to a lower level. An overall effect of LHRH-agonist treatment was observed for miR-133a and miR-133b expression $(P<$ $0.05)$, but not in miR-1 or miR-206 expression $(P>0.05)$. No significant interaction was observed in any of the four myomiRs, $(P>0.05)$. As mentioned in the introduction, testosterone may indirectly regulate myomiRs through increasing transcription of the MRFs MYF5, MYOD, and MYOG and the androgen receptor (AR) (Chen et al., 2006; Sweetman et al., 2008; Wannenes et al., 2008; Dubois et al., 2012; Nasipak and Kelley, 2012). We therefore measured mRNA levels of these genes at rest. Intriguingly, while most of the transcription factors remained unchanged the expression of MYOG was significantly higher in the LHRH-treated patients (Figure 3E).

miR-133a AND miR-133b ARE DOWN-REGULATED IN CASTRATED MICE As our human model included subjects with cancer and were lacking appropriate controls, we were concerned about non-specific 
Association between MyomiR expression in muscle and $\mathrm{VO}_{2}$ max in men and women

A

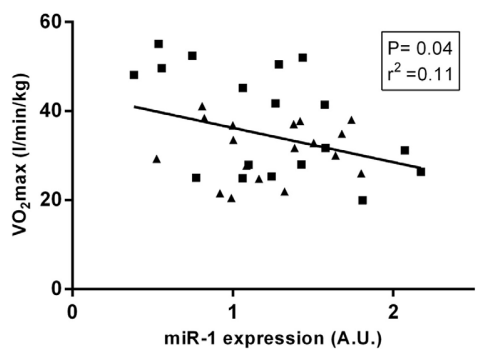

C

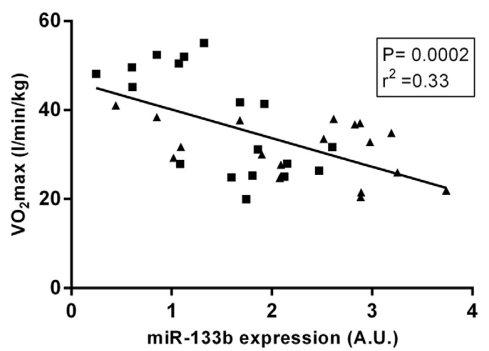

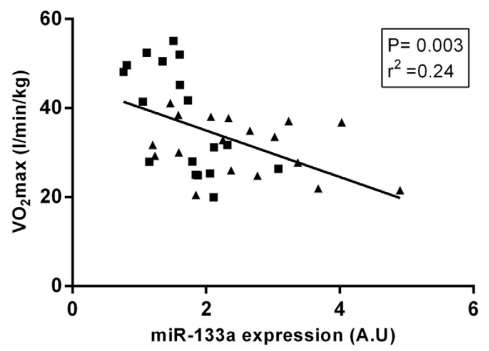

D

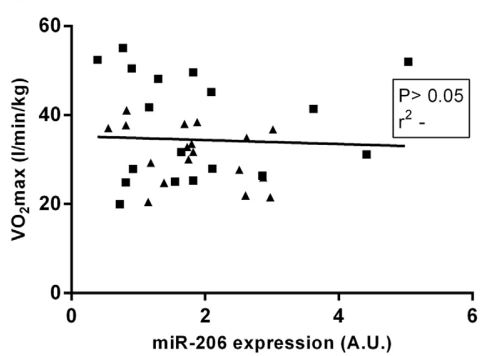

Association between MyomiR expression in muscle and testosterone levels in men

E

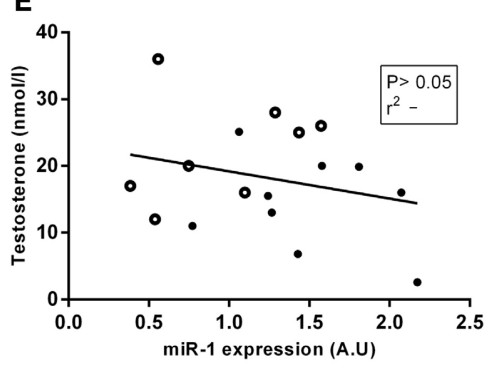

G

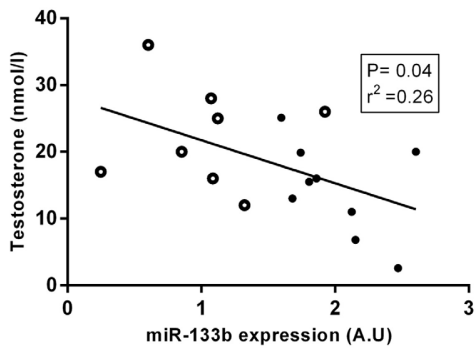

FIGURE 2 | Linear regression analysis between myomiR expression and physiological characteristics. The cohort described in Figure $\mathbf{1}$ was assessed for associations between muscular miRNA expression and physiological characteristics. In panel (A-D) are men symbolized (一) and women are symbolized ( $\mathbf{v})$. In panel (D,E) observations from elderly men are symbolized (•) and observations from younger men are symbolized (O). miR-1, miR-133a, and miR-133b (A-C) were

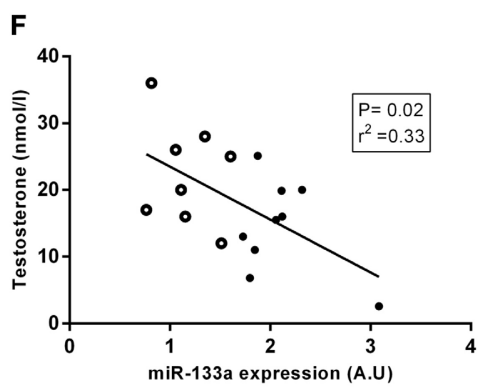

H

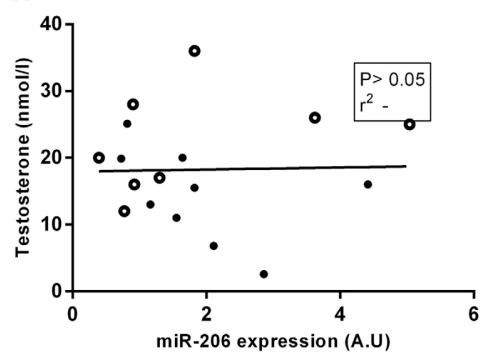

inversely correlated with maximal oxygen uptake in women and men ( $n=36) \quad\left(P<0.05,0.01\right.$ and 0.001, $R^{2}=0.11,0.24$, and 0.33). In addition, miR-133a (F) and miR-133b (G) expression were negatively correlated with testosterone levels in men $(n=18)(P<0.05$, $R^{2}=0.33$ and $\left.P<0.05, R^{2}=0.26\right) . \operatorname{miR}-1 \quad$ (E) and miR-206 (H) expression were not correlated with testosterone in men. Data are mean \pm s.e.m. effects. Therefore, we also measured myomiR expression in skeletal muscle of castrated mice, which eliminated circulating testosterone, and sham operated control mice. Consistent with our findings in the LHRH-agonist treated men with low circulating testosterone, castrated mice had a lower expression of mir-133a $(P<0.05)$ and mir-133b $(P<0.001)$ (Figure 4A). The expression of mir-1 and mir-206 were identical in skeletal muscle in both castrated and sham operated mice $(P>$ 

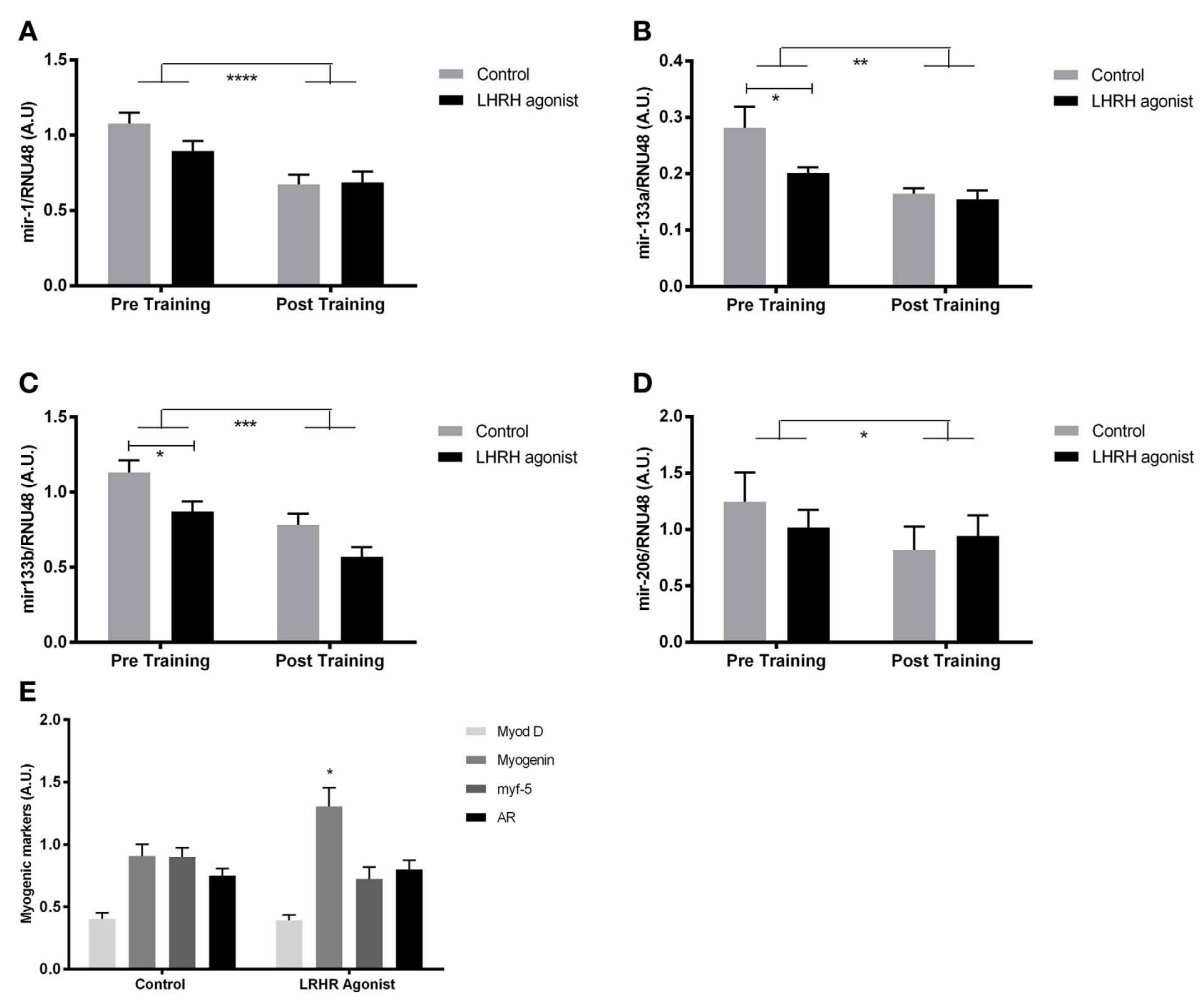

FIGURE 3 | MyomiR expression in testosterone depleted men before and after training. MyomiR expression in the skeletal muscle of LHRH agonist treated prostate cancer patients $(n=8)$ and healthy controls $(n=8)$, in resting samples prior to and following 12 weeks of aerobic exercise training. A Two-Way ANOVA (RM) (miR-1, ${ }^{* * * *} P<0.0001$. miR-133a, ${ }^{* *} P<0.01$. miR-133b, ${ }^{* * *} P<0.001$, miR-206 $\left.{ }^{*} P<0.05\right)$.

Bonferroni multiple comparison post-hoc tests revealed a significant lower

expression of mir-133a (B) $(* P=0.02)$ and mir-133b (C) $(* P=0.03)$, but not mir-1 (A) or miR-206 (D) in testosterone blocked patients at rest before training. The expression of myomiRs in testosterone blocked patients and healthy controls were identical after the training period. The expression of MYOG (E) $\left({ }^{*} P<0.05\right)$ at rest was upregulated in testosterone-blocked participants. No regulation of MYOD, MYF-5, or AR was detected. Data are mean \pm SEM.

0.05). Furthermore, the MRFs measured in castrated mice were unchanged with the exception of increased Myog mRNA levels, in line with the finding in our testosterone blocked human participants $(P<0.05)$ (Figure 4B).

\section{$5 \alpha$-DIHYDROTESTOSTERONE REGULATES miR-133a, miR-133b, AND miR-206 EXPRESSION IN HUMAN PRIMARY MYOCYTES}

To evaluate if testosterone directly regulates myomiR expression in skeletal muscle we established a cell culture model using human myotubes. Human myotubes were incubated with $100 \mathrm{nM}$ of testosterone or $100 \mathrm{nM} 5 \alpha$-dihydrotestosterone ( $5 \alpha$-DHT), which has a higher AR affinity than testosterone (Saartok et al., 1984). $5 \alpha$-DHT is formed in vivo by $5 \alpha$-reductase acting on testosterone intracellularly in myocytes (Sato et al., 2008). Whether testosterone is converted to $5 \alpha-\mathrm{DHT}$ in vitro is unknown, and thus we incubated primary myotubes with either testosterone or $5 \alpha$-DHT for $24 \mathrm{~h}$ (Sato et al., 2008). Consistent with the in vivo experiments suggesting a role for testosterone in regulating miR-133a/b, $5 \alpha$-DHT incubation in culture increased miR-133a and miR-133b $(P<0.05)$ (Figure 5A). Surprisingly, incubation with $5 \alpha-\mathrm{DHT}$ decreased miR-206 $(P<0.05)$, While MYF-5 was upregulated in response to testosterone or $5 \alpha$-DHT $(P<0.05)$, AR, MYOG, and MYOD mRNA was not significantly altered (Figure 5B).

\section{DISCUSSION}

In the current study, we found that the expression of miR1, miR-133a, and miR-133b was higher in the skeletal muscle of elderly compared to younger men and that miR-133a/b was higher expressed in women compared to men. miR-133a/b was inversely correlated with an age-dependent decrease of testosterone in men. However, our subsequent studies demonstrated that testosterone positively regulated miR-133a/b. Finally, our data suggest that increased physical activity compensates for the loss of testosterone in terms of myomiR regulation.

Our collective data from three independent models, indicate that testosterone up-regulates miR-133a, and 133b. Importantly, we also show that physical activity overrides the regulatory effect of testosterone on miR-133a/b expression. Our findings suggest that an age-dependent decline in testosterone and increase of miR-133a/b expression are independent events. It has been established that increased physical fitness is associated with a down-regulation of myomiRs (Nielsen et al., 2010; Keller et al., 2011). Therefore, it is likely that the decline in physical activity is the main determining factor involved in the age-dependent up-regulation of miR-1 and miR-133a/b.

The observed effects of testosterone and age as inducers of miR-133a and miR-133b expression are seemingly in opposition. 


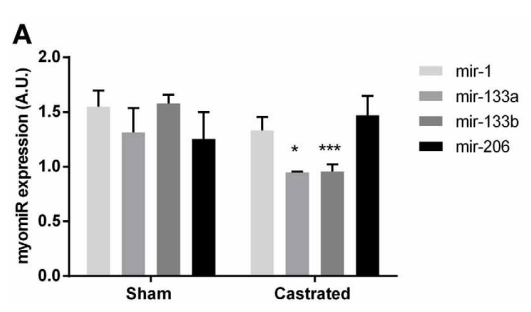

FIGURE 4 | Muscle of castrated $(n=5)$ and sham operated $(n=4)$ C57BL/6J mice. An unpaired t-test demonstrated a significant lower expression of mir-133a $\left({ }^{*} P<0.05\right)$ and mir-133b $\left({ }^{* * *} P<0.001\right)$ in the skeletal muscle of castrated mice. The expression of mir-1 and

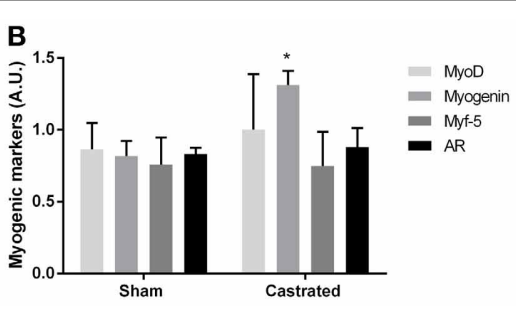

mir-206 were not significantly different between castrated and sham operated mice (A). Whereas the expression of Myog was upregulated $(* P<0.05)$ in castrated mice, no regulation of Myod, Myf-5, or Ar was detected $(P>0.05)$ (B). Data are mean \pm SEM.

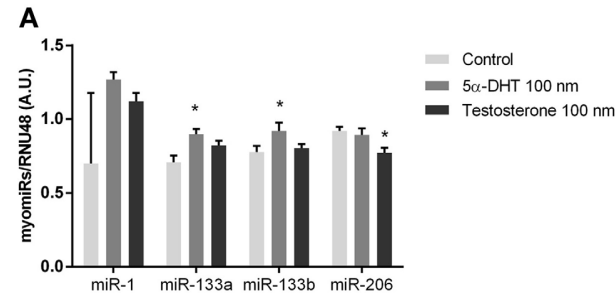

FIGURE 5 | myomiR expression in testosterone treated human muscle cells. myomiR expression in differentiated human primary myocytes in response to $100 \mathrm{nM}$ testosterone $(n=4)$, and $5 \alpha$-DHT $(n=4)$. Control cultures $(n=4)$ were added DMSO in same concentrations as testosterone and $5 \alpha$-DHT stimulated myocytes. miR-133a/b was upregulated in response

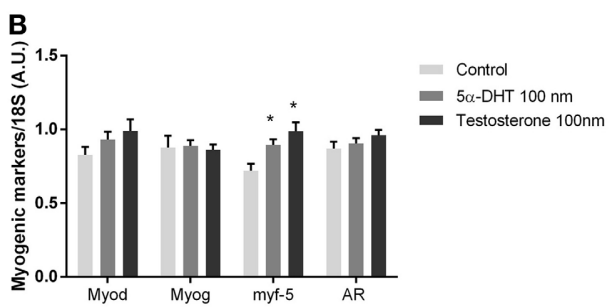

to $5 \alpha$-DHT treatment and mirR-206 expression was downregulated in response to to testosterone treatment $(* P<0.05)$ (A). Whereas the expression of Myf-5 was upregulated in response to $5 \alpha$-DHT and testosterone $(* P<0.05)$ no regulation of Myod, Myog or AR was detected (B). Data are mean \pm SEM.
However, in addition to miR-133a/b, miR-1 was induced in the elderly group. miR-1 has a specific set off predicted mRNA targets and the regulatory net-effect will thus differ from the effect of testosterone-mediated miRNA regulation. It is therefore possible that myomiR alterations contribute to age-dependent decline in muscle function. Interestingly, our human data suggests that the effects of testosterone on myomiR regulation are minor compared to the effect of increased physical activity. Androgenic control of miR-133a/b expression therefore seems to be a separate regulatory mechanism that may play a role during certain physiological conditions, such as physical inactivity.

Studies have shown that alterations in myomiR expression are associated with both hypertrophy and atrophy in skeletal muscle (Care et al., 2007; McCarthy and Esser, 2007). Importantly, age related loss of muscle mass has been extensively described in the literature, including the age intervals for the subjects in the current study (Forbes and Reina, 1970). However, our elderly subjects were healthy and we did not observe any age related differences in lean body mass within each gender group in the current study. Therefore, changes in myomiR expression might represent an early event preceding potential loss in muscle mass. Noteworthy, lean body mass is assumed to reflect the muscle mass of the subjects but this assumption is subject to some degree of uncertainty. A study by Loon and co-workers revealed that muscle cross sectional area and muscle fiber type size were markedly decreased in older subjects compared to young. However, differences in lean body mass between young and old when performing DXA scan measurements, was not detectable (Nilwik et al., 2013). Determination of lean body mass by DXA is an indirect quantification method and not a direct measurement of the actual muscle mass or cross sectional area, two physiological parameters directly related to muscle function. Therefore, it cannot be excluded that our older participants in fact have suffered from a loss of muscle function that we were not able to detect in the present cross-sectional study. In contrast to the age dependent up-regulation of myomiRs in men we did not observe a similar regulation pattern in women (Figure 1). A possible explanation for this could be less distinct differences in aerobic fitness between the elderly and younger women. Interestingly, the women had a markedly increase in miR-133a and miR-133b expression compared to men. Emphasizing the gender differences of skeletal muscle a recent study by Liu and co-workers demonstrated that the global transcriptome in muscle of older men and women differed markedly for groups of genes important for muscle function (Liu et al., 2013).

A few previous studies have investigated the age dependent miRNA alteration in human skeletal muscle. Global miRNA expression in the skeletal muscle of young vs. elderly men was compared using a miRNA microarray by Drummond et al. (2011). Drummond found that 18 miRNAs, including miR$133 \mathrm{a}$ and miR-133b, were differentially expressed. In contrast to our results from the current study, their microarray data pointed toward a downregulation of miR-133a/b in elderly men. Interestingly, Drummond et al. have also reported that the 
precursors to mature miRNAs, pri-miR-1, and pri-miR-133a, but not the mature forms, were increased in skeletal muscle of elderly men compared to younger controls (Drummond et al., 2008b). A number of reasons could explain the discrepancy with our data. In contrast to the study by Drummond et al. the testosterone levels in the current study differed between young and elderly men (Drummond et al., 2008a). Furthermore, aerobic fitness levels were not determined and our young subject group is approximately 8 years younger than in the study performed by Drummond and co-workers (Drummond et al., 2008a; Nielsen et al., 2010).

It is well known that miRNAs frequently are co-expressed in clusters with neighboring miRNAs (Baskerville and Bartel, 2005). However, in the current study we observe a co-regulation of myomiRs from separate loci in response to alterations in circulating testosterone levels. Interestingly, steroid hormonal posttranscriptional control of miRNA expression in several cell types, including muscle, has been described in the literature (Yamagata et al., 2009). Thus, it is possible that the regulation of mir-133a/b expression by testosterone occurs through a post-transcriptional processing of the pri- and/or pre-miRNA transcripts.

Our human participants depleted for testosterone were recently diagnosed with prostate cancer and on LHRH agonist therapy. Some limitations with this model exist and should be discussed. For instance, each subject was not his own control since ethics dictated that treatment was started immediately after the diagnosis excluding that we could apply a longitudinal study design. Furthermore, differences between the control and treated group could be due to the cancer rather than the lack of circulating testosterone. Therefore, we included two additional models to verify our findings from the human in vivo study; castration of male mice which also eliminates circulating testosterone and stimulation of human myotubes in vitro. These two models both confirmed our initial findings in the human in vivo model. We thus show a physiological role of testosterone in the regulation of miR-133a and miR-133b in human and murine skeletal muscle.

In this study we have examined the myomiR expression in resting skeletal muscle of elderly and younger men and women along with the role of testosterone and physical fitness on muscle myomiR regulation. We clearly show that alterations in testosterone levels are sufficient to alter myomiR expression. We furthermore conclude that aerobic exercise training is likely to independently regulate myomiR and seem to override any effects of testosterone.

\section{AUTHOR CONTRIBUTIONS}

Study conception and design: Søren Nielsen, Matthew J. Laye, Bente K. Pedersen. Collection, analysis and interpretation of data: Søren Nielsen, Matthew J. Laye, Bente K. Pedersen, Thine Hvid, Camilla Scheele, Meghan Kelly, Christine Dethlefsen, Neha Mathur, Kamilla Winding, Birgitte Lindegaard. Drafting the article or revising it critically for important intellectual content:: Søren Nielsen, Matthew J. Laye, Bente K. Pedersen, Thine Hvid, Camilla Scheele, Meghan Kelly, Christine Dethlefsen, Neha Mathur, Kamilla Winding, Birgitte Lindegaard. All authors approved the final version for publication.

\section{ACKNOWLEDGMENTS}

The Centre of Inflammation and Metabolism (CIM) is supported by a grant from the Danish National Research Foundation (DNRF55). The Centre for Physical Activity Research (CFAS) is supported by a grant from Trygfonden. CIM is part of the UNIK Project: Food, Fitness, and Pharma for Health and Disease, supported by the Danish Ministry of Science, Technology, and Innovation. CIM is a member of DD2-the Danish Center for Strategic Research in Type 2 Diabetes (the Danish Council for Strategic Research, grant no. 09-067009 and 09-075724). The Copenhagen Muscle Research Centre (CMRC) is supported by a grant from the Capital Region of Denmark.

\section{REFERENCES}

Baek, D., Villen, J., Shin, C., Camargo, F. D., Gygi, S. P., and Bartel, D. P. (2008). The impact of microRNAs on protein output. Nature 455, 64-71. doi: 10.1038 /nature07242

Bartel, D. P. (2004). MicroRNAs: genomics, biogenesis, mechanism, and function. Cell 116, 281-297. doi: 10.1016/S0092-8674(04)00045-5

Baskerville, S., and Bartel, D. P. (2005). Microarray profiling of microRNAs reveals frequent coexpression with neighboring miRNAs and host genes. RNA 11, 241-247. doi: 10.1261/rna.7240905

Booth, F. W., and Zwetsloot, K. A. (2010). Basic concepts about genes, inactivity and aging. Scand. J. Med. Sci. Sports 20, 1-4. doi: 10.1111/j.1600-0838.2009.00972.x

Care, A., Catalucci, D., Felicetti, F., Bonci, D., Addario, A., Gallo, P., et al. (2007). MicroRNA-133 controls cardiac hypertrophy. Nat. Med. 13, 613-618. doi: $10.1038 / \mathrm{nm} 1582$

Chen, J. F., Mandel, E. M., Thomson, J. M., Wu, Q., Callis, T. E., Hammond, S. M., et al. (2006). The role of microRNA-1 and microRNA-133 in skeletal muscle proliferation and differentiation. Nat. Genet. 38, 228-233. doi: 10.1038/ ng1725

Delic, D., Grosser, C., Dkhil, M., Al-Quraishy, S., and Wunderlich, F. (2010). Testosterone-induced upregulation of miRNAs in the female mouse liver. Steroids 75, 998-1004. doi: 10.1016/j.steroids.2010.06.010

Deslypere, J. P., and Vermeulen, A. (1984). Leydig cell function in normal men: effect of age, life-style, residence, diet, and activity. J. Clin. Endocrinol. Metab. 59, 955-962. doi: 10.1210/jcem-59-5-955

Drummond, M. J., Dreyer, H. C., Pennings, B., Fry, C. S., Dhanani, S., Dillon, E. L., et al. (2008a). Skeletal muscle protein anabolic response to resistance exercise and essential amino acids is delayed with aging. J. Appl. Physiol. 1985 104, 1452-1461. doi: 10.1152/japplphysiol.00021.2008

Drummond, M. J., McCarthy, J. J., Fry, C. S., Esser, K. A., and Rasmussen, B. B. (2008b). Aging differentially affects human skeletal muscle microRNA expression at rest and after an anabolic stimulus of resistance exercise and essential amino acids. Am. J. Physiol. Endocrinol. Metab. 295, E1333-E1340. doi: 10.1152/ajpendo.90562.2008

Drummond, M. J., McCarthy, J. J., Sinha, M., Spratt, H. M., Volpi, E., Esser, K. A., et al. (2011). Aging and microRNA expression in human skeletal muscle: a microarray and bioinformatics analysis. Physiol. Genomics 43, 595-603. doi: 10.1152/physiolgenomics.00148.2010

Dubois, V., Laurent, M., Boonen, S., Vanderschueren, D., and Claessens, F. (2012). Androgens and skeletal muscle: cellular and molecular action mechanisms underlying the anabolic actions. Cell. Mol. Life Sci. 69, 1651-1667. doi: 10.1007/s00018-011-0883-3

Forbes, G. B., and Reina, J. C. (1970). Adult lean body mass declines with age: some longitudinal observations. Metabolism 19, 653-663. doi: 10.1016/00260495(70)90062-4

Frederiksen, L., Hojlund, K., Hougaard, D. M., Brixen, K., and Andersen, M. (2012). Testosterone therapy increased muscle mass and lipid oxidation in aging men. Age (Dordr.) 34, 145-156. doi: 10.1007/s11357-011-9213-9

Gallagher, I. J., Scheele, C., Keller, P., Nielsen, A. R., Remenyi, J., Fischer, C. P., et al. (2010). Integration of microRNA changes in vivo identifies novel molecular features of muscle insulin resistance in type 2 diabetes. Genome Med. 2, 9. doi: $10.1186 /$ gm 130

Gibney, J., Wolthers, T., Johannsson, G., Umpleby, A. M., and Ho, K. K. (2005). Growth hormone and testosterone interact positively to enhance protein and 
energy metabolism in hypopituitary men. Am. J. Physiol. Endocrinol. Metab. 289, E266-E271. doi: 10.1152/ajpendo.00483.2004

Heemers, H. V., and Tindall, D. J. (2007). Androgen receptor (AR) coregulators: a diversity of functions converging on and regulating the AR transcriptional complex. Endocr. Rev. 28, 778-808. doi: 10.1210/er.2007-0019

Huang, M. B., Xu, H., Xie, S. J., Zhou, H., and Qu, L. H. (2011). Insulin-like growth factor-1 receptor is regulated by microRNA-133 during skeletal myogenesis. PLoS ONE 6:e29173. doi: 10.1371/journal.pone.0029173

Hvid, T., Winding, K., Rinnov, A., Dejgaard, T., Thomsen, C., Iversen, P., et al. (2013). Endurance training improves insulin sensitivity and body composition in prostate cancer patients treated with androgen deprivation therapy. Endocr. Relat. Cancer 20, 621-632. doi: 10.1530/ERC-12-0393

Keller, P., Vollaard, N. B., Gustafsson, T., Gallagher, I. J., Sundberg, C. J., Rankinen, T., et al. (2011). A transcriptional map of the impact of endurance exercise training on skeletal muscle phenotype. J. Appl. Physiol. 110, 46-59. doi: 10.1152/japplphysiol.00634.2010

Kelly, D. M., and Jones, T. H. (2013). Testosterone: a metabolic hormone in health and disease. J. Endocrinol. 217, R25-R45. doi: 10.1530/JOE-12-0455

Lim, L. P., Lau, N. C., Garrett-Engele, P., Grimson, A., Schelter, J. M., Castle, J., et al. (2005). Microarray analysis shows that some microRNAs downregulate large numbers of target mRNAs. Nature 433, 769-773. doi: 10.1038/ nature 03315

Liu, D., Sartor, M. A., Nader, G. A., Pistilli, E. E., Tanton, L., Lilly, C., et al. (2013). Microarray analysis reveals novel features of the muscle aging process in men and women. J. Gerontol. A Biol. Sci. Med. Sci. 68, 1035-1044. doi: 10.1093/ gerona/glt015

Lujambio, A., and Lowe, S. W. (2012). The microcosmos of cancer. Nature 482, 347-355. doi: 10.1038/nature10888

McCarthy, J. J., and Esser, K. A. (2007). MicroRNA-1 and microRNA-133a expression are decreased during skeletal muscle hypertrophy. J. Appl. Physiol. 102, 306-313. doi: 10.1152/japplphysiol.00932.2006

Muller, M., den Tonkelaar, I., Thijssen, J. H., Grobbee, D. E., and van der Schouw, Y. T. (2003). Endogenous sex hormones in men aged 40-80 years. Eur. J. Endocrinol. 149, 583-589. doi: 10.1530/eje.0.1490583

Nasipak, B., and Kelley, D. B. (2012). Developing laryngeal muscle of Xenopus laevis as a model system: androgen-driven myogenesis controls fiber type transformation. Dev. Neurobiol. 72, 664-675. doi: 10.1002/dneu.20983

Nielsen, S., Scheele, C., Yfanti, C., Akerstrom, T., Nielsen, A. R., Pedersen, B. K., et al. (2010). Muscle specific microRNAs are regulated by endurance exercise in human skeletal muscle. J. Physiol. 588, 4029-4037. doi: 10.1113/jphysiol.2010.189860

Nilwik, R., Snijders, T., Leenders, M., Groen, B. B., van, K. J., Verdijk, L. B., et al. (2013). The decline in skeletal muscle mass with aging is mainly attributed to a reduction in type II muscle fiber size. Exp. Gerontol. 48, 492-498. doi: 10.1016/ j.exger.2013.02.012

Phillips, B. E., Hill, D. S., and Atherton, P. J. (2012). Regulation of muscle protein synthesis in humans. Curr. Opin. Clin. Nutr. Metab. Care 15, 58-63. doi: 10.1097/MCO.0b013e32834d19bc

Ramamani, A., Aruldhas, M. M., and Govindarajulu, P. (1999). Differential response of rat skeletal muscle glycogen metabolism to testosterone and estradiol. Can. J. Physiol. Pharmacol. 77, 300-304. doi:10.1139/y99-016

Rao, P. K., Kumar, R. M., Farkhondeh, M., Baskerville, S., and Lodish, H. F. (2006). Myogenic factors that regulate expression of muscle-specific microRNAs. Proc. Natl. Acad. Sci. U.S.A. 103, 8721-8726. doi: 10.1073/pnas.0602831103

van Rooij, E., Liu, N., and Olson, E. N. (2008). MicroRNAs flex their muscles. Trends Genet. 24, 159-166. doi: 10.1016/j.tig.2008.01.007

Ringholm, S., Bienso, R. S., Kiilerich, K., Guadalupe-Grau, A., AachmannAndersen, N. J., Saltin, B., et al. (2011). Bed rest reduces metabolic protein content and abolishes exercise-induced mRNA responses in human skeletal muscle. Am. J. Physiol. Endocrinol. Metab. 301, E649-E658. doi: 10.1152/ajpendo.00230.2011

Russell, A. P., Wada, S., Vergani, L., Hock, M. B., Lamon, S., Leger, B., et al. (2012). Disruption of skeletal muscle mitochondrial network genes and miRNAs in amyotrophic lateral sclerosis. Neurobiol. Dis. 49C, 107-117. doi: 10.1016/j.nbd. 2012.08.015

Saartok, T., Dahlberg, E., and Gustafsson, J. A. (1984). Relative binding affinity of anabolic-androgenic steroids: comparison of the binding to the androgen receptors in skeletal muscle and in prostate, as well as to sex hormone-binding globulin. Endocrinology 114, 2100-2106. doi: 10.1210/endo-114-6-2100

Sato, K., Iemitsu, M., Aizawa, K., and Ajisaka, R. (2008). Testosterone and DHEA activate the glucose metabolism-related signaling pathway in skeletal muscle. Am. J. Physiol. Endocrinol. Metab. 294, E961-E968. doi: 10.1152/ajpendo.00678.2007

Scheele, C., Nielsen, S., Kelly, M., Broholm, C., Nielsen, A. R., Taudorf, S., et al. (2012). Satellite cells derived from obese humans with type 2 diabetes and differentiated into myocytes in vitro exhibit abnormal response to IL-6. PLoS ONE 7:e39657. doi: 10.1371/journal.pone.0039657

Schiaffino, S., and Mammucari, C. (2011). Regulation of skeletal muscle growth by the IGF1-Akt/PKB pathway: insights from genetic models. Skelet. Muscle 1, 4. doi: 10.1186/2044-5040-1-4

Sweetman, D., Goljanek, K., Rathjen, T., Oustanina, S., Braun, T., Dalmay, T., et al. (2008). Specific requirements of MRFs for the expression of muscle specific microRNAs, miR-1, miR-206 and miR-133. Dev. Biol. 321, 491-499. doi: 10.1016/j.ydbio.2008.06.019

Torjesen, P. A., and Sandnes, L. (2004). Serum testosterone in women as measured by an automated immunoassay and a RIA. Clin. Chem. 50, 678-679. doi: 10.1373/clinchem.2003.027565

Wannenes, F., Caprio, M., Gatta, L., Fabbri, A., Bonini, S., and Moretti, C. (2008). Androgen receptor expression during C2C12 skeletal muscle cell line differentiation. Mol. Cell. Endocrinol. 292, 11-19. doi: 10.1016/j.mce.2008.05.018

Wyce, A., Bai, Y., Nagpal, S., and Thompson, C. C. (2010). Research resource: the androgen receptor modulates expression of genes with critical roles in muscle development and function. Mol. Endocrinol. 24, 1665-1674. doi: 10.1210/me.2010-0138

Yamagata, K., Fujiyama, S., Ito, S., Ueda, T., Murata, T., Naitou, M., et al. (2009). Maturation of microRNA is hormonally regulated by a nuclear receptor. $\mathrm{Mol}$. Cell 36, 340-347. doi: 10.1016/j.molcel.2009.08.017

Yarasheski, K. E. (2003). Exercise, aging, and muscle protein metabolism. J. Gerontol. A Biol. Sci. Med. Sci. 58, M918-M922. doi: 10.1093/gerona/58.10. M918

Yasui, T., Matsui, S., Tani, A., Kunimi, K., Yamamoto, S., and Irahara, M. (2012). Androgen in postmenopausal women. J. Med. Invest. 59, 12-27. doi: 10.2152/jmi.59.12

Zhao, Y., Ransom, J. F., Li, A., Vedantham, V., von Drehle, M., Muth, A. N., et al. (2007). Dysregulation of cardiogenesis, cardiac conduction, and cell cycle in mice lacking miRNA-1-2. Cell 129, 303-317. doi: 10.1016/j.cell.2007.03.030

Conflict of Interest Statement: The authors declare that the research was conducted in the absence of any commercial or financial relationships that could be construed as a potential conflict of interest.

Received: 20 September 2013; accepted: 15 December 2013; published online: 23 January 2014.

Citation: Nielsen S, Hvid T, Kelly M, Lindegaard B, Dethlefsen C, Winding K, Mathur $N$, Scheele C, Pedersen BK and Laye MJ (2014) Muscle specific miRNAs are induced by testosterone and independently upregulated by age. Front. Physiol. 4:394. doi: 10.3389/ fphys.2013.00394

This article was submitted to Striated Muscle Physiology, a section of the journal Frontiers in Physiology.

Copyright (c) 2014 Nielsen, Hvid, Kelly, Lindegaard, Dethlefsen, Winding, Mathur, Scheele, Pedersen and Laye. This is an open-access article distributed under the terms of the Creative Commons Attribution License (CC BY). The use, distribution or reproduction in other forums is permitted, provided the original author(s) or licensor are credited and that the original publication in this journal is cited, in accordance with accepted academic practice. No use, distribution or reproduction is permitted which does not comply with these terms. 